\title{
Decomposing TIAM-MACRO to Assess Climatic Change Mitigation
}

\author{
Socrates Kypreos • Antti Lehtila
}

Received: 13 April 2014 / Accepted: 26 February 2015 / Published online: 23 April 2015

(C) Springer International Publishing Switzerland 2015

\begin{abstract}
TIAM-MACRO (TM) is a mathematical programming growth model where the global multi-region bottom-up engineering model TIAM is linked with a topdown macroeconomic module MACRO to maximize an inter-temporal utility function for a single representative producer-consumer agent in each region. The size of TM is such that non-linear (NL) optimal solutions cannot be obtained even when the best available personal computers and solvers are used. Therefore, an alternative is proposed based on decomposition methods converting TM to a small-size NL macroeconomic model, called TIAMMACRO Stand-Alone (TMSA), and where the energy model TIAM is substituted by appropriate quadratic costsupply functions (QSF). The TIAM model and the TMSA are calibrated to the demands estimated with a scenario generator and are then solved iteratively. This report concentrates on the description and foundation of the algorithm and explains why an adjusted production function is needed to allow for sectoral income and price elasticities that reproduce/calibrate the baseline scenario. It is shown that the decomposed problem for a single region is calibrated and solved to exactly the same results as the original problem in $3 \mathrm{~min}$ of computer time instead of 2-3 h without decomposition. Also, for the first time, we are able to solve the global TM model with 15 regions in $1.5 \mathrm{~h}$ applying the approach based on TMSA (in Windows 7, 64-bit workstation, solution in a single thread).
\end{abstract}

\footnotetext{
S. Kypreos $(\bowtie)$

Energy Economics Group, Paul Scherrer Institut,

Villigen, Switzerland

e-mail: Socrateskypreos@gmail.com

A. Lehtila

VTT Technical Research Centre of Finland, Espoo, Finland

e-mail: antti.lehtila@vtt.fi
}

Keywords Climate change $\cdot$ Macroeconomic impacts . Hybrid models $\cdot$ Engineering models $\cdot$ Decomposition methods

\section{Introduction}

The representation of the interactions between the energy, economy, and environment has been one of the key motivations in model development in the last decades. The world challenges of two energy crises, together with the environmental issues of climate change and the expected depletion of fossil fuel resources, inspired the development of appropriate technological fixes together with methods that simulate mathematically the interactions and feedbacks between energy, economy, and environment for policy assessments and recommendations. As expected, different methods have been developed, classified as optimization or computable general equilibrium models. Our work is focusing on integrated energy-economy models with a comprehensive representation of the energy system. The interested reader is recommended to consult also recent publications $[1,2]$ discussing integrated assessment models for climate policy analysis and a comprehensive review of relatively recent developments in the field of integrated assessment models [3].

It has been demonstrated [4] that "computable general equilibrium models (CGE) following the Arrow-Debreu [5] equilibrium theory can also be obtained from global (economy-wide) optimization that implements Pareto optimality and uses the equilibrium characterization introduced by Negishi". An example of such optimization models isMARKAL-MACRO [6] of the IEA-Energy Technology Systems Analysis Project (ETSAP) family of models, which has the form of mathematical programming and is a hybrid optimization equilibrium model. According to Capros et al. 
[4], the computable and the optimization equilibrium models are equivalent as the former, represented as a system of simultaneous equations, corresponds to the first-order optimum conditions of the mathematical programming problem and includes prices as model variables. Prices as variables enable a straightforward representation of policy instruments and market-related institutional characteristics and are easily solvable while large-scale non-linear programming (NLP) optimization models are difficult to solve.

According to the classification described by Böhringer and Rutherford [7], the TM model is a simple hybrid model. The authors also proposed to use the mixed complementary format to represent in the same mathematical formulation the process oriented technological models like The Integrated MARKALEFOM System (TIMES) with a multi-agent and multi-sectoral general equilibrium model of households, government, and firms and the interplay of the primary production factors. Although the authors give some pedagogic examples of their approach, such generalized formulations linking top-down with bottom-up models are not yet developed. Nevertheless, quite a few efforts are undertaken to integrate, based on soft linking methods, TIMES with general equilibrium models like, e.g., GEMINI-E3 [8], GEM-E3 [9], in order to capture sectoral impacts in terms of welfare and labor losses as function of stringent environmental policies ${ }^{1}$.

The MARKAL-MACRO model and its latest incarnation of TIMES-MACRO have been developed as part of the Implementing Agreement of the IEA-ETSAP to assess, among others, the whole energy system and climate change mitigation options and policies on the regional, national, or global level. They establish a link between the process engineering models MARKAL [10] or TIME $\mathrm{S}$ [11] and the macroeconomic module MACRO. MACRO maximizes an inter-temporal utility function for a single representative producer-consumer agent. Key variables in this module are production factors such as capital stock, labor, and energy services, which together determine the total output of the economy based on their relative prices. The module obtains an endogenous specification of demands for energy services as function of economic activity and relative prices, the specification of substitution effects between the production factors, and the assessment of the macroeconomic implications of energy and environmental policies. The module is a reduced form representation of economic equilibria with a help of production functions. The consequence of the non-linear

\footnotetext{
${ }^{1}$ The link of such models with TIMES goes in two directions; first, the CGE model is used to generate a consistent set of drivers which together with income and price elasticities define demands for energy services. Then, a TIMES solution returns final energy flows per industrial sectors, households, services, and transportation, over-writing the Leontief relations of the CGE model until all sectorial markets clear and equilibrium prices are defined.
}

functions used to represent the economy and the climate module is the excessive computer time needed to find an optimal solution.

Although large-scale versions of multi-regional models like RMARKAL or TIMES integrated assessment model (TIAM) [11-13] linked and driven by regional MACROeconomic modules have been generated in the past, one had difficulties to solve them even when the most powerful commercial solvers and state-of-the-art computers are used. This situation appears already with the medium-size multi-regional global MARKAL-MACRO (GMM) trade model $[14,15]$ with five world regions and becomes even worse when the other versions of the global regionalized MARKAL (RMARKAL) or TIAM models are used that have a detailed regional representation of 15 world regions or more. On the other hand, the well-known MESSAGE-MACRO model of International Institute for Applied Systems Analysis (IIASA) [16] is an established but similar model assessing global scenarios of climate change mitigation. This large-scale model is successfully and efficiently solved by decomposition methods. In this paper, we describe the formulation and solution of TIAMMACRO following a decomposition and iterative approach similar to MESSAGE-MACRO. Another effort to define decomposition methods solving multi-regional MARKAL-MACRO models with global trade has been undertaken by Büeler [17], described also in [18], but it has taken hours of computing time to converge with the consequence that the method was not followed further in the ETSAP community.

In summary, this paper formulates the TIAM-MACRO model as mathematical programming problem and applies decomposition methods converting the model to an equivalent macroeconomic module called TIAM-MACRO Stand-Alone (TMSA) where the energy model TIAM is substituted by appropriate quadratic cost-supply functions (QSF). The TIAM model and TMSA are calibrated to the demands of a scenario generator and solved iteratively.

The rest of the report is organized as follows: A simple scenario generator (SG) is described in Section 2; Section 3 explains the calibration procedure where the demand decoupling factors (ddf) introduced in TMSA reproduce the demand developments for the baseline case; Section 4 defines the first single region TMSA model, followed by an example where the baseline and the $\mathrm{CO}_{2}$-constrained cases are solved using the algorithm; Section 5 explains the multi-regional TMSA, the Negishi [19] welfare function, and the iterative procedure based on the sequential equilibrium algorithm of Rutherford [20] while we discuss the precision obtained and the computing time needed to solve the global and multi-regional model under the carbon constraint. Finally, conclusions are given in the last section. 


\section{The Scenario Generator}

The scenario generator describes a scenario-storyline quantifying key socioeconomic developments like population, economic growth, lifestyle changes, energy intensity, policies to favor technological innovations and global diffusion of technological change, degree of environmental policy legislation introduced, and the dynamics towards urbanization (megacities) and globalization versus decentralized developments. Based on these scenario assumptions and eventually the use of computable general equilibrium models for checking the internal consistency of the socioeconomic assumptions, TIAM specifies the time-dependent demand for energy services. Solving the partial equilibrium engineering model TIAM, we satisfy the equilibrium demand for energy services and their prices and complete the description of the energy system development in consistency with the socioeconomic assumptions and the technological options that correspond to the scenario-storyline as represented in the database of TIMES.

The ETSAP family of models defines demands that reflect past trends and exogenous assumptions on population, GDP, energy intensity, and technology penetration based on demand drivers and their elasticities. As most of the efficiency improvement options are included in the database of the engineering model explicitly, the specific selection of autonomous efficiency improvement factors (aeeif) applied below reflects mainly lifestyle changes. A simple but useful relation for demand projections will be the following:

$\frac{D_{i t}}{D_{i 1}}=\left[\frac{\mathrm{dr}_{i t}}{\mathrm{dr}_{i 1}}\right]^{\alpha_{i}} \cdot\left[\frac{P_{i t}}{P_{i 1}}\right]^{-\sigma_{i}} \cdot \prod_{\tau=1, t}\left(1-\text { aeeif }_{i \tau}\right)^{\mathrm{ypp}_{\tau}}$

Here, $D_{i t}$ is the demand projection for commodity (sector) $i$ and period $t ; D_{i 1}$ is the same demand for the initial year of analysis calibrated to energy statistics for the socioeconomic assumptions and representative efficiencies of the end-use devices valid for this year; $\mathrm{dr}_{i t}$ is the demand driver; $\alpha_{i}$ is the driver elasticity; $\sigma_{i}$ is the price elasticity; aeeif ${ }_{i t}$ is the autonomous efficiency improvement factor per demand commodity; $\mathrm{ypp}_{\tau}$ is the years per period; and $\frac{P_{i t}}{P_{i 1}}$ is the index of relative price of demand for commodity $i$. TIAM assumes different growth rates and elasticities of demand drivers for each individual demand category. Usually, some consistency checks of economic assumptions and the demand projections generated based on Eq. 1 must be completed. If for some regions the calibration is not feasible for the same growth rates as the one assumed, this indicates the existence of a basic inconsistency in key assumptions and the need for revision. This is done with the help of a growth model like MERGE [21] or with a CGE model like GEM-E3. IIASA for example adjusts projections to the results of MERGE while ETSA $\mathrm{P}$ is using GEM-E3.

\section{The Calibration Method}

Next, we discuss the procedure applied to calibrate TM to the demand projections and the macroeconomic assumptions for both, the initial year and the time-dependent developments such that energy statistics of the starting year together with the demand and GDP projections of the scenario generator are reproduced. Notice that only when this work is concluded successfully, the model is ready to assess the implication of environmental policies like, e.g., a carbon constraint.

\subsection{Benchmarking the Production Function}

The production function applied in TM is a constant elasticity of substitution (CES) function between the value-added pairs capital $K$ and labor $l$ and the demands for energy services $D$ to describe the economic output production $Y$, with $\alpha$ being the capital value share. The CES production function allows substitution between the pair capital-labor and the energy services when the relative prices change, (the time index is dropped for simplicity):

$$
\begin{aligned}
Y= & {\left[a\left(K^{\alpha} \cdot l^{(1-\alpha)}\right)^{\rho}+\sum_{i} b_{i} \cdot D_{i}^{\rho}\right]^{1 / \rho} } \\
& \text { the CES elasticity is } \sigma \quad \text { with } \rho \\
= & (\sigma-1) / \sigma \quad \sigma \neq 0,1
\end{aligned}
$$

Applying the first-order optimality condition for the starting year, we are able to benchmark the parameters $a$ and $b_{i}$ of this function and to derive the implicit demand function which is valid when the maximization conditions are satisfied. The unit change of the economic output per unit of energy services is the price of services $P_{i t}$, and this defines the implicit demand function:

$D_{i t}=Y_{t} \cdot\left(P_{i t} / b_{i}\right)^{-\sigma}$

With known values of demands and their prices from the TIMES solution as well as the initial values of the macroeconomic activities, one is able to benchmark the coefficients of the production function.

\subsection{Demand Decoupling Factors}

The implicit demands for energy services based on Eq. 3 depend on the economic output, the price per unit of 
services $P_{i t}$, and the CES elasticity of substitution and have a unitary income elasticity not able to distinguish for any structural change in economic growth and apply the same "price" elasticity for all demand categories. A flexible production function can be specified in TM to overcome these drawbacks when one takes into consideration some annual demand decoupling factors (ddf) for each demand category, e.g.:

$\mathrm{DM}_{i t}=D_{i t} \cdot \prod_{\tau=1, t}\left(1-\mathrm{ddf}_{i \tau}\right)^{\mathrm{ypp}} \equiv D_{i t} \cdot F_{i t}$

The variable $\mathrm{DM}_{i t}$ refers to the demand constraints of the energy model while $D_{i t}$ is the primary input factors of production associated with the energy services. Finally, the production function adopted in TM assumes a factoraugmenting coefficient ddf that represents priceindependent technological progress like efficiency improvement as it decouples energy demand from economic growth. The coefficients of the adopted production function can be estimated applying the first-order optimality conditions in a CES formulation that explicitly considers the ddf and the demand constraints of the energy model TM:

$$
\begin{aligned}
& Y_{t}=\left[a\left(K_{t}^{\alpha} \cdot l_{t}^{(1-\alpha)}\right)^{\rho}+\sum_{i} b_{i} \cdot\left(\mathrm{DM}_{i t} / F_{i t}\right)^{\rho}\right]^{1 / \rho} \\
& \quad \text { and if } f_{t} \equiv a\left(K_{t}^{\alpha} \cdot l_{t}^{(1-\alpha)}\right)^{\rho}+\sum_{i} b_{i} \cdot\left(\mathrm{DM}_{i t} / F_{i t}\right)^{\rho} \\
& \quad \text { then } Y=f^{1 / \rho}
\end{aligned}
$$

Applying the first-order optimality condition for each period, we get an implicit demand function able to benchmark the equation at the starting year first and then the ddf. The implicit demand function is as follows:

$\mathrm{DM}_{i t}=F_{i t}^{1-\sigma} \cdot Y_{t} \cdot\left(P_{i t} / b_{i}\right)^{-\sigma}$

With known constants $b_{i}$, one can specify the factor $a$ based on Eq. 5 as in the first year $l_{1}=1$ and $F_{i 1}=1$, then

$a=\left[Y_{1}^{\rho}-\sum_{i} b_{i} \cdot\left(\mathrm{DM}_{i 1}\right)^{\rho}\right] /\left(K_{1}^{\alpha \cdot \rho}\right)$

In the following section, we describe how TM can be disaggregated into an energy model (i.e., TIMES) and a macroeconomic module called TIMES-MACRO Stand-Alone (TMSA) and explain how the ddf are calibrated.

\section{TIMES-MACRO Stand-Alone}

We explain the decoupling of TM into the linear energy model TIMES and the NLP MACRO starting first with a single region model before going to the more complex regionalized global model with trade. In the subsequent model formulations, we accept the energy demand projections of a scenario generator related to the socioeconomic development and all other assumptions underlying the baseline case. Then, a set of ddf consistent to this development will be defined and the decomposition approach will be explained.

\subsection{The Specification of TMSA}

The TMSA model includes only the macroeconomic (non-linear) part of TM. Energy demand is usually a function of price and income, $D_{i t}=f\left(P_{i t}, Y_{t}\right)$, but we apply a quadratic supply-cost function to correlate demand to the energy cost $\mathrm{EC}_{t}$ substituting for the full energy model representation. The TMSA problem for a single region is defined by maximizing the utility function $U$ of the region:

Max $U=\sum_{t=1, T} \operatorname{dfact}_{t} \cdot \ln C_{t} \quad$ that subjects to the following constraints :

$Y_{t}=\left[a\left(K_{t}^{\alpha} \cdot l_{t}^{(1-\alpha)}\right)^{\rho}+\sum_{i} b_{i} \cdot D_{i t}{ }^{\rho}\right]^{1 / \rho} \quad$ production function,

$Y_{t}=C_{t}+I_{t}+\mathrm{EC}_{t} \quad$ use of output

$$
\begin{aligned}
K_{t} & =(1-\delta)^{\mathrm{yp}_{t-1}} K_{t-1} \\
& +\frac{1}{2}\left[\mathrm{ypp}_{t} \cdot I_{t}+\mathrm{ypp}_{t-1} \cdot I_{t-1}(1-\delta)^{\mathrm{ypp}_{t-1}}\right] \quad \text { capital formation }
\end{aligned}
$$

$K_{T} \cdot\left(\operatorname{gr}_{T}+\delta\right) \leq I_{T} \quad$ terminal condition

$\mathrm{EC}_{t}=\mathrm{qa}_{t}+\sum_{i} \mathrm{qb}_{i t} \cdot \mathrm{DM}_{i t}^{2} \quad$ quadratic supply function

$\mathrm{DM}_{i t}=D_{i t} \cdot \prod_{\tau=1, t}\left(1-\mathrm{ddf}_{i \tau}\right)^{\mathrm{ypp}_{\tau}} \quad$ demand decoupling function 
where

$C_{t} \quad$ Annual consumption in period $t$ (variable)

$Y_{t} \quad$ Annual production in period $t$ (variable)

$K_{t} \quad$ Total capital in period $t$ (variable)

$I_{t} \quad$ Annual investments in period $t$ (variable)

$\mathrm{DM}_{i t} \quad$ Annual demand in TIMES for commodity $i$ in period $t$ (variable)

$D_{i t} \quad$ Annual energy service demand in MACRO for commodity $i$ in period $t$ (variable)

$\mathrm{EC}_{t} \quad$ Annual energy system costs in MACRO in period $t$ (variable)

dfact $_{t}$ Discount factor per period for the utility function

$a \quad$ Production function constant (benchmarked)

$b_{i} \quad$ Demand coefficient for demand commodity $i$ (benchmarked)

$\operatorname{ddf}_{i t} \quad$ Demand decoupling factor for demand commodity $i$ in period $t$ (calibration parameter)

$\operatorname{ypp}_{t} \quad$ Number of years per period in period $t$

$\delta \quad$ Capital depreciation rate

$\mathrm{gr}_{t} \quad$ Growth rate in period $t$ (calibration parameter)

$\alpha \quad$ Capital value share

$l_{t} \quad$ Annual labor growth index in period $t$ (exogenous parameter)

$\mathrm{qa}_{t} \quad$ Constant term of the quadratic supply-cost function (derived from TIMES)

$\mathrm{qb}_{i t} \quad$ Coefficient for demand $i$ of the quadratic supply-cost function (derived from TIMES)

The TIMES demands $\mathrm{DM}_{i t}$ and the equilibrium price $P_{i t}$ established in the solution of the partial equilibrium version of the model describe the reference development and are correlated with the production function demands for energy services as defined in Eq. 14:

$\mathrm{DM}_{i t}=D_{i t} \cdot \prod_{\tau=1, t}\left(1-\operatorname{ddf}_{i \tau}\right)^{\mathrm{ypp}} \equiv D_{i t} \cdot F_{i t}$

while the implicit demand function given in Eq. 6 can be approximated using labor growth instead of the economic output:

$\mathrm{DM}_{i t}=Y_{t} \cdot F_{i t}^{1-\sigma} \cdot\left(P_{i t} / b_{i t}\right)^{-\sigma} \cong l_{t} \cdot F_{i t}^{1-\sigma} \cdot\left(P_{i t} / b_{i}\right)^{-\sigma}$

This equation allows for an initial approximation of the parameters $F_{i t}$ (or $d d f$ ) in TIMES in agreement with the projections of the scenario generator as demands, labor growth, and prices are known, once the model is solved. Therefore, an iterative procedure is needed to estimate the equilibrium ddf and to calibrate the baseline demands to the demands of the scenario generator that was first introduced in Kypreos [22] and is described by Remme and Blesl [23] in details.
In TMSA, we substitute the full-scale TIMES model representing the $\mathrm{EC}_{t}$ as a quadratic energy supply function (Eq. 13) of demands such that two key variables of TMSA are correlated to each other. As the energy system of both models should be the same at the end of the iterations, the partial derivative of $\mathrm{EC}_{t}$ in respect to demands should be equal to the shadow prices of demands $P_{t i}$ in TIMES. $\partial \mathrm{EC}_{t} / \partial D i=$ $P_{i t}=2 \cdot \mathrm{qb}_{i t} \cdot \mathrm{DM}_{i t}$ while the coefficients of Eq. 13 are related to the activities of a TIMES solution as follows:

$\mathrm{qb}_{i t}=\frac{P_{i t}}{2 \cdot \mathrm{DM}_{i t}}$ and $\mathrm{qa}_{t}=\mathrm{EC}_{t}-\sum_{i} \mathrm{qb}_{i t} \cdot \mathrm{DM}_{i t}^{2}$

With all input data known, the TMSA model is solved iteratively. The reporting routine after solving TMSA provides new demands and labor growth and the growth of the gross economic output. These parameters are used to determine a new set of ddf and the QSF. With these new ddf, the QSF, and the new labor growth rates, we reformulate the TMSA model and a new run starts. This sequential execution of TIMES and TMSA models adjusting demands and the ddf continues until the demands calculated in TMSA sufficiently match the specified demands in TIMES and the GDP growth estimated in TMSA matches the user-specified GDP projection. The number of iterations in the calibration routine can be controlled by a tolerance error while the NLP solution is rapidly converging as the size of the model is small.

\subsection{First Applications of the Algorithm with MARK AL-MACRO}

We have proposed and applied for the first time the approach using the MARKAL-MACRO (MM) model of Switzerland [24] solving the model either directly or with the MACRO Stand-Alone (MSA) version comparing the differences in the obtained results. The first iteration with MSA for the baseline case gives GDP errors in the order of $2 \%$, but adjusting for the labor growth rates, we have an excellent model calibration for the GDP development. The relative GDP error was below 10-4, while the computation time is a fraction of seconds.

After calibrating the baseline, we rerun the partial equilibrium MARKAL assuming for example a $20 \%$ reduction of carbon emissions and return to MSA. For that run, we keep unchanged the calibration constants of the CES function, the labor productivity, and the ddf as in the baseline but we define a new cost-supply function to represent the equilibrium of the energy system under the carbon constraint. Then, the MSA is re-solved to estimate the GDP reduction. This is shown in Fig. 1 concluding that the quadratic cost function approximates well the energy system behavior and the GDP losses. 
Fig. 1 GDP change relative to baseline in percent for a carbon emission reduction constraint of $20 \%$ below the 1990 's emission levels. There is an increasing error over time in MSA due to light differences in discounting methods between the two versions of the models applied at that time

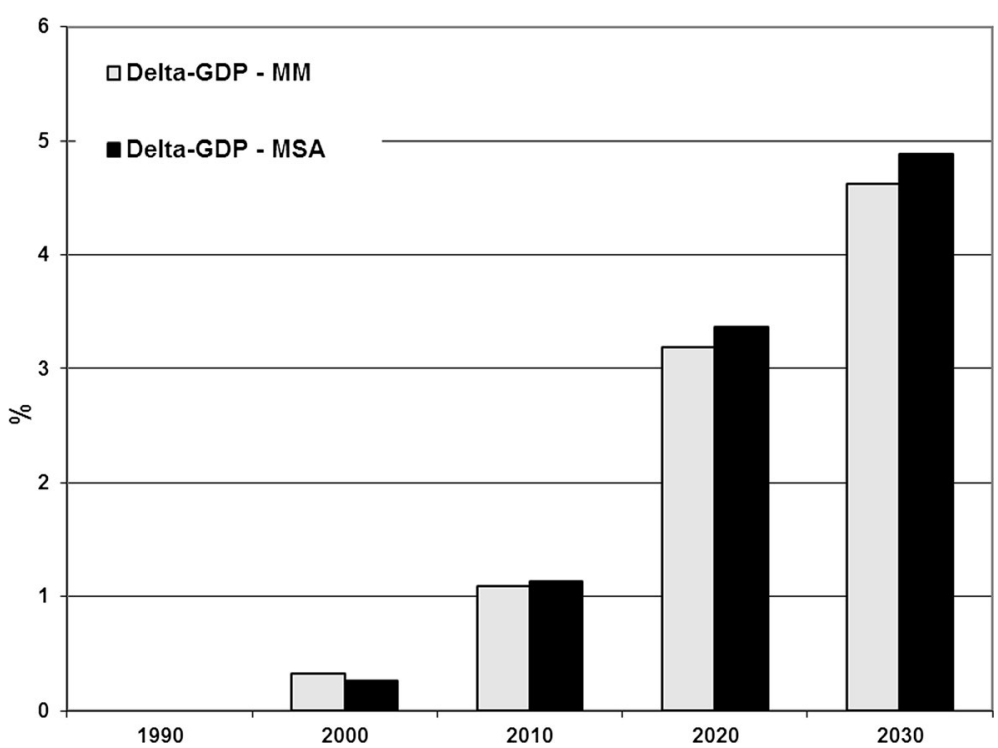

\subsection{Recent Applications of the Algorithm for TM}

After the formulation of the NLP multi-regional model of TM, we have tested the algorithm for a single region (USA) comparing computing times and solution accuracy. Test results indicate that TMSA is able to identify the same solution as TM being more than 100 times faster. The calibration run has taken less than 1 min solved with the new TMSA algorithm versus $150 \mathrm{~min}$ for the full TM model. Solving a $\mathrm{CO}_{2}$ cumulative constrained case has taken $2 \mathrm{~min}$ for TMSA versus $200 \mathrm{~min}$ for TM. Thus, it takes 3 min CPU time with a modern (but not a premium PC) to solve the overall constrained problem (calibration and a policy case) versus $350 \mathrm{~min}$ without applying the decomposition method. This computer time requirements explain also the unpopularity of TM model among the ETSAP users. Figure 2 presents the errors in terms of GDP and the GDP differences between the two solution approaches for the baseline and the policy case. The calibration error for the GDP in the baseline case is below 10-8. The error for the GDP differences remains as shown in Fig. 2 and is for sure well below 10-4 in both bases. Notice that this level of accuracy was obtained after adjusting some discounting discrepancies between TIMES linear programming (LP) and TMSA.

\section{Extension to Multi-regional TIAM-SA Model}

In the following, we decompose the global and multi-regional macroeconomic growth model TIAM-MACRO into a multiregional partial equilibrium energy problem, e.g., TIAM and a multi-regional macroeconomic model called TMSA maximizing the global welfare function.

\subsection{The TIAM-MACRO Stand-Alone Formulation}

For the new stand-alone MACRO formulation, the original MACRO had to be generalized to support multiple regions. In the multi-regional case, the model is solved by maximizing the Negishi-weighted sum of regional utilities, iterating between the stand-alone TM model (TMSA) and the standard TIAM model. The TMSA model explicitly considers only the trade of the numéraire good, as the trade in all energy products is defined in the TIAM model. The formulation of the stand-alone MACRO implementation, applied in the decomposition approach, can be described with the following equations:

$\operatorname{Max} U=\sum_{t=1}^{T} \sum_{r} \mathrm{nwt}_{r} \cdot \mathrm{pwt}_{t} \cdot$ dfact $_{r, t} \cdot \ln \left(C_{r, t}\right)$

$Y_{r, t}=C_{r, t}+I_{r, t}+\mathrm{EC}_{r, t}+\mathrm{NTX}(\mathrm{nmr})_{r, t}$

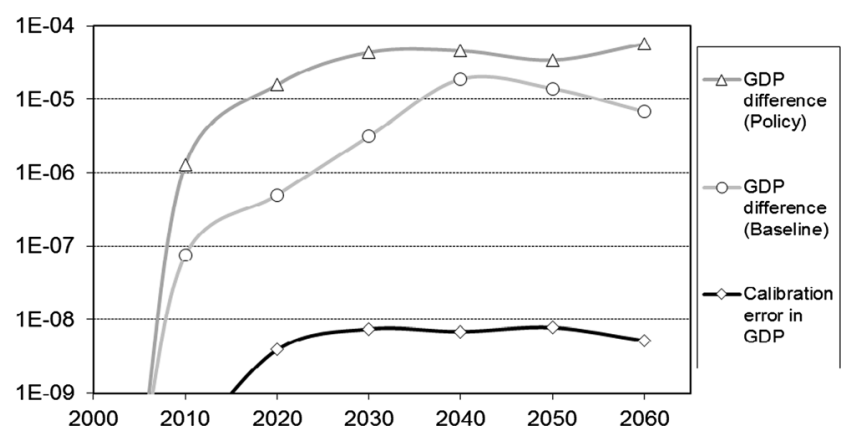

Fig. 2 Errors in GDP calibration and in GDP differences for the baseline and the policy case obtained for the single region TM model of the USA 


$$
\begin{aligned}
& Y_{r, t}=\left(a_{r} \cdot K_{r, t}^{\alpha_{r} \cdot \rho_{r}} \cdot l_{r, t}^{\left(1-\alpha_{r}\right) \rho_{r}}+\sum_{i} b_{r, i} \cdot \mathrm{DM}_{r, t, i}^{\rho_{r}}\right)^{\frac{1}{\rho_{r}}} \\
& K_{r, t+1}=\left(1-\delta_{r}\right)^{\mathrm{ypp}_{t}} \cdot K_{r, t} \\
& +\frac{1}{2}\left[\operatorname{ypp}_{t} \cdot\left(1-\delta_{r}\right)^{\mathrm{ypp}_{t}} \cdot I_{r, t}+\mathrm{ypp}_{t+1} \cdot I_{r, t+1}\right] \\
& K_{r, T} \cdot\left(\operatorname{gr}_{r, T}+\delta_{r}\right) \leq I_{r, T} \\
& \mathrm{DT}_{r, t, i}=\operatorname{aeeifac}_{r, t, i} \cdot \mathrm{DM}_{r, t, i} \\
& \mathrm{EC}_{r, t}=\mathrm{qa}_{r, t}+\sum_{k} \mathrm{qb}_{r, t, k} \cdot\left(\mathrm{DT}_{r, t, i}\right)^{2}+\mathrm{amp}_{r, t} \\
& \sum_{r} \operatorname{NTX}(\mathrm{nmr})_{r, t}=0 \quad \forall\{t\}
\end{aligned}
$$

\begin{tabular}{|c|c|}
\hline$C_{r, t}$ & Annual consumption in period $t$ (variable) \\
\hline$Y_{r, t}$ & Annual production in period $t$ (variable) \\
\hline$K_{r, t}$ & Total capital in period $t$ (variable) \\
\hline$I_{r, t}$ & Annual investments in period $t$ (variable) \\
\hline $\mathrm{DM}_{r, t, i}$ & $\begin{array}{l}\text { Annual demand in MACRO for commodity } i \\
\text { in period } t \text { (variable) }\end{array}$ \\
\hline $\mathrm{DT}_{r, t, i}$ & $\begin{array}{l}\text { Annual demand in TIMES for commodity } i \text { in } \\
\text { period } t \text { (variable) }\end{array}$ \\
\hline $\mathrm{EC}_{r, t}$ & $\begin{array}{l}\text { Annual energy system costs in MACRO in } \\
\text { period } t \text { (variable) }\end{array}$ \\
\hline $\operatorname{NTX}(\mathrm{nmr})_{r, t}$ & $\begin{array}{l}\text { Net exports of the numéraire good for region } r \text {, } \\
\text { in time period } t \text { (variable) }\end{array}$ \\
\hline$a_{r}$ & Production function constant for region $r$ \\
\hline$\alpha_{r}$ & Capital value share in region $r$ \\
\hline & $\begin{array}{l}\text { Corrects for the annualized investment cost of } \\
\text { existing capacities in starting period }\end{array}$ \\
\hline$b_{r, i}$ & $\begin{array}{l}\text { Demand coefficient for demand } \\
\text { commodity } i\end{array}$ \\
\hline
\end{tabular}

$$
l_{r, 1}=1 \quad \text { and } \quad l_{r, t+1}=l_{r, t} \cdot\left(1+\operatorname{growv}_{r, t}\right)^{\frac{\mathrm{yp}_{t}+\mathrm{ypp}_{t+1}}{2}}
$$

where

aeeifac $_{r, t, i} \quad$ Autonomous energy efficiency improvement factor

$\operatorname{ddf}_{r, \tau, i}$
$\delta_{r}$
dfact $_{r, t}$
$\operatorname{gr}_{r, T}$
$l_{r, t}$
nwt $_{r}$
pwt $_{t}$

qa $_{r, t}$
$\mathrm{qb}_{r, t, i}$
ypp $_{t}$
$\rho_{r}$
$T$

Demand decoupling factor (calibration parameter)

Capital depreciation rate per region

Utility discount factor for period $t$ and region $r$

Growth rate in last period and region $r$ (calibration parameter)

Annual labor growth index in period $t$ and region $r$

Negishi weight for region $r$ (defined iteratively)

Period-length-dependent weights in the utility function (these multipliers are introduced in cases where the period lengths are not equal)

Constant term per period of the quadratic supply-cost function Coefficient for demand commodity $i$ in the quadratic supply-cost function, period $t$

Number of years per period in period $t$

Substitution constant

Number of periods in the model horizon

The primary differences in relation to the standard MACRO formulation are the following:

- The use of Negishi weights in the objective function when the model is multi-regional. Negishi iterations balance for inter-temporal discounted trade deficits of a region over the full time horizon of the analysis;

- The inclusion of trade of the numéraire good NTX $(\mathrm{nmr})_{r, t}$ in the production function;

- The introduction of the trade balance of the numéraire good (Eq. 24);

- The replacement of the full TIAM LP cost accounting by quadratic supply-cost functions for each demand commodity (Eq. 23).

\subsection{The Standard TIMES LP Formulation}

The second part of the decomposed model, the TIAM LP model, uses the standard TIAM formulation, which can be written in short as follows:

Min NPV $=\sum_{r=1}^{R} \sum_{y \in T}\left(1+d_{r, y}\right)^{\mathrm{ref}-y} \cdot \mathrm{AEC}_{r, y}$

$A \cdot x=b$ and $x \geq 0$ 
where

NPV Net present value of all energy system costs

$T \quad$ The set of years within the model horizon

ref Reference year for discounting

$d_{r, y} \quad$ Capital discount factor for region $r$ in year

$\mathrm{AEC}_{r, y} \quad$ Annual energy system cost in region $r$ and year $y$

$A \quad$ Coefficient matrix for all other model equations

$x \quad$ Vector of all model variables

$b \quad$ RHS constant vector for all other model equations

$R \quad$ Number of internal regions in the model

For a comprehensive treatment of the standard TIAM LP formulation, see Loulou et al. (2005). In order to make the LP formulation more analogous with the MACRO objective function, the objective function of the standard TIAM code can be rewritten in terms of period-wise average annual costs and period-specific discount factors, as follows:

Min NPV $=\sum_{r=1}^{R} \sum_{t=1}^{T} \operatorname{pvf}_{r, t} \cdot \mathrm{EC}_{r, t}$

where

$\mathrm{pvf}_{r, t}$ Present value factor for period $t$ in region $r$

$\mathrm{EC}_{r, t}$ Period-wise average annual energy system costs in region $r$ and period $t$

$T \quad$ Number of periods $t$ in the model horizon

\subsection{General Specifications of the Decomposition Algorithm}

\subsubsection{Algorithm for Baseline Calibration}

In both MACRO formulations, the use of the MACRO mode for evaluating policy scenarios requires that the demand decoupling factors (ddf) and labor growth rates have first been calibrated with the baseline scenario and the corresponding GDP growth projections. The core part of the calibration procedure is the updating of the demand decoupling factors and labor growth rates between successive iterations of the calibration algorithm.

In the TMSA implementation, all the basic mathematical formulas for updating the demand decoupling factors and labor growth rates are fully equivalent to those in the standard TM formulation and have been explained previously and are therefore omitted here. The reader is advised to consult the documentation of Remme and Blesl [23] for the details on those parts. The overall algorithm for calibrating the baseline is given below:
- Step 1: Solve the baseline TIAM LP model:

(a) Minimize the partial equilibrium LP objective function: $\operatorname{Min} \mathrm{ObjZ}=\sum_{r, t} \mathrm{pvf}_{r, t} \cdot \mathrm{EC}_{r, t}$

(b) Calculate quadratic supply-cost functions QSF for the demands

- Step 2: Solve the stand-alone MACRO model (TMSA):

(c) Calculate new ddf and labor growth rates (d) Maximize the total utility: $U=\sum_{r, t} \mathrm{pwt}_{t} \cdot$ dfact $_{r t}$.
$\ln \left(Y_{r t}-I_{r t}-\mathrm{EC}_{r t}\right)$

(e) If max. error in demands and GDP are above tolerance, go to Step $2 \mathrm{c}$

- Step 3: If multi-regional, iterate TMSA with Negishi weights

(f) Calculate initial Negishi weights nwt $r_{r}$

(g) Solve TMSA with the modified objective:

$U=\sum_{r, t} \mathrm{nwt}_{r} \cdot \mathrm{pwt}_{t} \cdot \mathrm{dfact}_{r t} \cdot \ln \left(Y_{r t}-I_{r t}-\mathrm{EC}_{r t}-\mathrm{NTX}(\mathrm{nmr})_{r t}\right)$

(h) Calculate new nwt $r$, and if difference is above tolerance, update the ddf and labor growth rates, and go back to Step $3 \mathrm{~g}$

- Step 4: Write final calibration parameters into the data dictionary (DD) file of TIAM

\subsubsection{Algorithm for Policy Scenarios}

The main purpose of using TM is, of course, in running policy scenarios. The multi-regional TIMES-MSA model is solved by maximizing the Negishi-weighted sum of regional utilities while we iterate between the two models on the Negishi weights and demand levels in all periods, until they converge. The decomposition algorithm employed in TMSA for the policy scenarios can be outlined as follows:

- Step 1: Solve the policy scenario TIAM LP model:

(a) Minimize the partial equilibrium LP objective function: $\mathrm{Min} \mathrm{ObjZ}=\sum_{r, t} \mathrm{pvf}_{r, t} \cdot \mathrm{EC}_{r, t}$

(b) Calculate the QSF. Read the calibrated ddf and labor growth factors from the DD file that was saved when the calibration run was terminated.

- Step 2: Solve TMSA (with Negishi loop if multi-regional)

(c) Calculate initial Negishi weights nwt ${ }_{r}$

(d) Maximize the total utility $U: U=\sum \mathrm{nwt}_{r} \cdot \mathrm{pwt}_{t}$. dfact $_{r t} \cdot \ln \left(Y_{r t}-I_{r t}-\mathrm{EC}_{r t}-\mathrm{NTX}(\mathrm{nmr})_{r t}\right)^{r, t}$ 


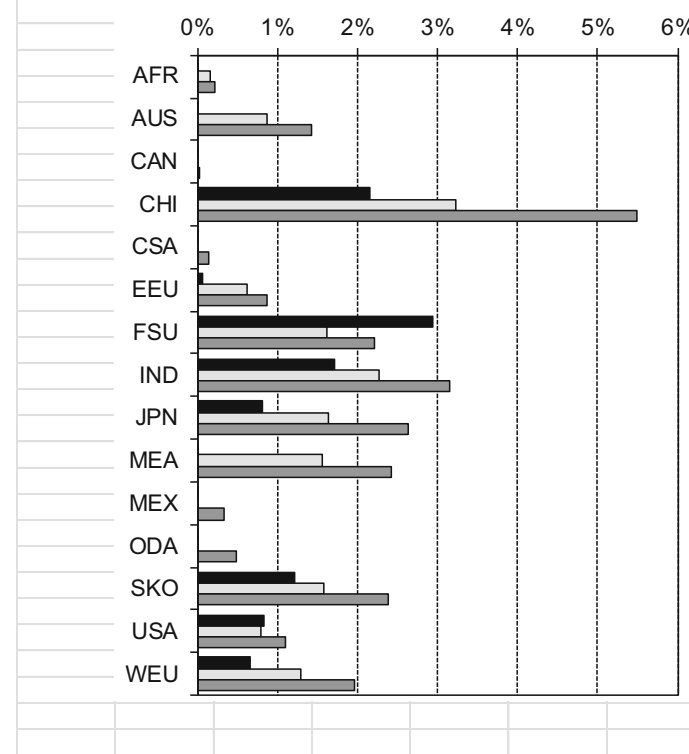

Fig. 3 Regional GDP losses for the constrained cases for a moderate (left) and a stringent policy case (right). The maximum losses take place in the fast growing and developing economies of China $(\mathrm{CHI}$

(e) Calculate the new nwt $r$, and if change is above tolerance, go to Step $2 \mathrm{~d}$

(f) If the error in demand levels is below tolerance, go to Step 3

(g) Update the LP demands according to the TMSA results and then resolve the standard TIMES model LP using the ObjZ objective

(h) Calculate new QSF and go back to Step 2d

- Step 3: Calculate all model results and finish

The initial Negishi weights are set as being proportional to the regional output share. To balance for intertemporal trade deficits, we need to properly adjust the weights in an iterative approach following the sequential optimization algorithm of Rutherford [20]. As the global net exports, per traded product trd, are balanced to zero at each time period, we can use the dual of this constraint to define the price of traded products $\pi_{\operatorname{trd}, t}$. The weights are adjusted using the normalized price of the traded products, the trade excess, and the inverse of the marginal regional utility, i.e.,

$$
\begin{aligned}
\mathrm{NW}_{r}= & \sum_{t, \mathrm{rrd}} \pi_{\mathrm{trd}, t} \cdot \mathrm{NTX}_{r, t, \mathrm{trd}} \\
& +\sum_{t} \pi_{\mathrm{nmr}, t} \cdot C_{r, t} \text { with } \mathrm{nwt}_{r} \\
= & \mathrm{NW}_{r} / \sum_{r} \mathrm{NW}_{r}
\end{aligned}
$$

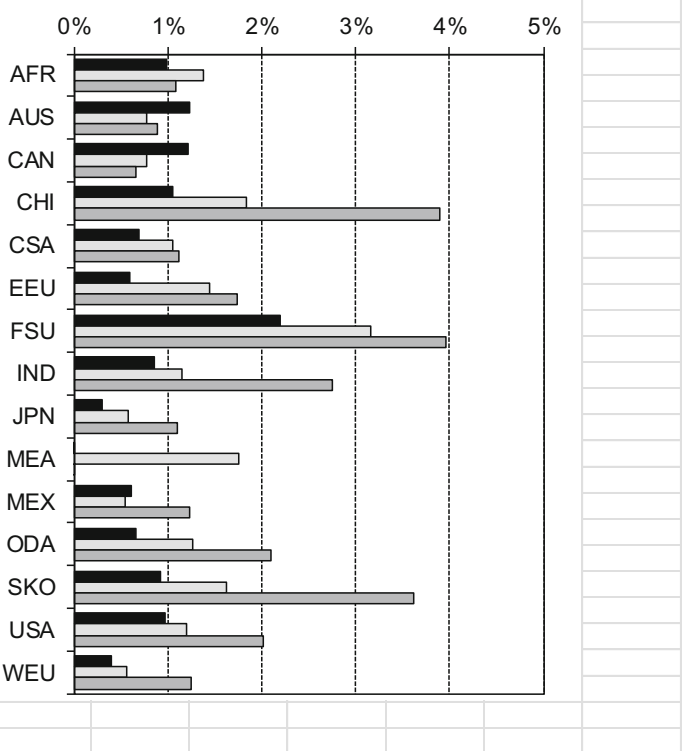

and India $(I N D)$ and South Korea $(S K O)$ and the energy-exporting region of the former Soviet Union (FSU)

According to Rutherford, if the weights are inversely proportional to the marginal utility per region, the solution is Pareto optimal.

\subsection{Climate Policy Test Runs}

Two scenarios have been estimated for testing the algorithm with a time horizon until 2060:

Case A: This case represents regional $\mathrm{CO}_{2}$ targets resembling the long-term pledges of the Copenhagen accord; various countries have committed in the COP15 and COP16 in Cancun.

Case B: A global target for radiative forcing is imposed that should correspond to a concentration limit of 550 ppm $\mathrm{CO}_{2}$ equivalent.

These cases have been solved with the global TIAMMACRO model, and the key results of regional GDP

Table 1 Performance of the TMSA algorithm (2005-2060)

\begin{tabular}{llllll}
\hline TIAM-MACRO & \multicolumn{2}{l}{ Model size } & & & \multicolumn{2}{c}{ Run time (min) } \\
\cline { 2 - 3 } \cline { 5 - 6 } Test model & Equations & Variables & & Calibration & Policy run \\
\hline TIAM-USA & 305,00 & 52,800 & & 1 & 2 \\
TIAM-6R & 199,800 & 508,300 & & 4 & 28 \\
TIAM-10R & 318,200 & 678,100 & & 7 & 59 \\
TIAM-15R & 457,600 & 864,000 & & 11 & 89 \\
\hline
\end{tabular}


losses versus the baseline are presented in the subsequent Fig. 3.

\subsection{Performance of the Algorithm}

A few runs with TMSA have been executed to test the performance and accuracy of the algorithm. The most significant test was the solution of TM for a single region as the code could be solved with both model formulations while results are directly comparable. The USA TMSA model validated well against $\mathrm{TM}$ as the solution was identical. Interesting is to report the computer time needed to solve the calibration and the policy analysis case of TMSA as function of the number of regions (see Table 1). As explained before, for a single region, the model is calibrated in less time than $1 \mathrm{~min}$ while a policy case takes 2 min, i.e., more than 100 times faster than TM. The calibration time is increased to 4 and $7 \mathrm{~min}$ when the number of regions increases to six and 15, respectively, while the policy cases need 28 and 89 min with a time horizon of 2005 to 2060. The modest increase in computing time which is almost linear with the number of regions is partly explained as the whole TIAM model was defined when solving the regional subset cases, with the excluded TIAM regions fixed to the levels of the full 15 region solution.

During 2014 the model has been applied in the ETSAP project imposing stringent concentration levels like those corresponding to the policy target of $2^{\circ} \mathrm{C}$ warming, confirming the accuracy and performance of the algorithm for different ranges of carbon emission reduction [25].

\section{Final Conclusions}

The large-scale general equilibrium growth model TM is solvable only when decomposed to the linear energy model TIAM and a non-linear macroeconomic stand-alone model (TMSA) where quadratic supply functions substitute for the energy system represented in TIAM. Associated with that problem is the need to develop a scenario generator that allows elaborating simple but consistent scenarios for TIAM.

We have presented herein the methodology that allows projecting demands for energy services for a postulated set of macroeconomic assumptions, lifestyle changes, and energy intensities with a help of a scenario generator based on sectoral drivers and income and price elasticities. Then, we explained why the demand decoupling factors (ddf) need to be defined for TIAM-MACRO as this model uses unitary income elasticity and the same elasticity of substitution across all sectors. The model can be calibrated such that the postulated GDP growth and the demands for energy services assumed in the scenario generator are reproduced in TMSA. This is done with a minimum investment in respect of computation time. We have also presented the theoretical foundation of this calibration as well as the decomposition algorithm and the execution times needed for low tolerance errors obtained during the calibration itself and when applying the model for a policy case. This is done for a single country model first and then, after the approach is extended to the multi-regional TM model, for the global and regionalized model.

Although the quadratic supply-cost function is a simple and approximate meta-model that substitutes for the full-scale supply model and the marginal costs for some demand categories are sensitive to small demand changes, the algorithm is able to give an exact calibration for the baseline case followed by good results for the carbon-constrained case as the tolerance error in demand evaluation is below $10-4$. The prerequisite for a successful application of the QSF is to have all the important and linearized constraints determining the restructuring of the energy system included in the linear model TIAM. This is because the quadratic formulation of these functions allows for small changes around the demand variables when searching for optimal solutions and converges in small steps. Finally, the single region TM model run is reduced to $2 \mathrm{~min}$ when decomposition is applied while the direct method for calibration and policy analysis needs a few hours of computer time. Also, for the first time, we are able to solve the global TIAM-MACRO model with 15 regions in $1.5 \mathrm{~h}$ applying the approach based on TMSA (in Windows 7, 64-bit workstation, solution in a single thread).

Acknowledgments The IEA/ETSAP support for the initiation and completion of the project and the trust of GianCarlo Tosato, Project Head of ETSAP, for his never-ending hope concerning the final success of this work, are highly appreciated.

\section{References}

1. Wilkerson, T. J., Leibowicz, D. B., Turner, D. D., \& Weyant, P. J. (2015). Comparison of integrated assessment models: carbon price impacts on U.S. energy. Energy Policy, 76, 18-31.

2. Kriegler, E., Petermann, N., Krey, V., Schwanitz, V. J., Luderer, G., Ashina, S., Bosetti, V., Eom, J., Kitous, A., Méjean, A., Paroussos, L., Sano, F., Turton, H., Wilson, C., \& Van Vuuren, D. P. (2014). Diagnostic indicators for integrated assessment models of climate policy. Technological Forecasting and Social Change. doi:10.1016/ j. techfore.2013.09.020.

3. Ortiz, R. A., Markandya, A. (2009). Integrated impact assessment models with an emphasis on damage functions: a literature review. BC3 Working Paper Series 2009-06. Bilbao: Basque Centre for Climate Change (BC3). http://www.bc3research.org/lits publications.html.

4. Capros, P., Georgakopoulos, T., Van Regemorter, D., Proost, S., Schmidt, T., \& Conrad, K. (1997). The GEM-E3 model for the European Union. Journal of Economic \& Financial Modelling, 4(2\&3), 51-160.

5. Arrow, K. J., \& Debreu, G. (1954). Existence of an equilibrium for a competitive economy. Econometrica, 22, 265-290. 
6. Manne, A., \& Wene, C.-O. (1992). MARKAL-MACRO: a linked model for energy-economy analysis. No. BNL-47161. Upton: Brookhaven National Lab.

7. Böhringer, C., \& Rutherford, T. F. (2005). Integrating bottom-up into top-down: a mixed complementarity approach. ZEW Discussion Papers 05-28. ZEW - Zentrum für Europäische Wirtschaftsforschung / Center for European Economic Research.

8. Labriet, M., Loulou, R., Vielle, M., \& Drouet, L. (2008). Coupling TIAM and GEMINI-E3. Proceedings of the ETSAP Meeting, SophiaAntipolis, December 17, 2008.

9. Simões, S., Fortes, P., Seixas, J., van Regemorter, D., \& Ferreira, F. (2013). Top-down and bottom-up modelling to support low carbon scenarios: climate policy, implications. Climate Policy Journal, 13(3), 1-20. doi:10.1080/14693062.2013.768919.

10. Fishbone, L. G., \& Abilock, H. (1981). Markal, a linear-programming model for energy systems analysis: technical description of the BNL version. International Journal of Energy Research, 5(4), 353-375.

11. Loulou, R., Remne, U., Kanudia, A., Lehtila, A., \& Goldstein, G. (2005). Documentation for the TIMES Model (Part I, II and III), Energy Technology Systems Analysis Programme (ETSAP). http:// www.etsap.org/tools.htm.

12. SAGE (2003). Office of integrated analysis and forecasting, energy information administration. Washington, DC: U.S. Department of Energy. The System for Analysis of Global Energy markets (SAGE) model of the US-EIA, DOE/EIA-M072 (2003)/1, August 2003. http://www.eia.doe.gov/bookshelf/docs.html.

13. Haurie, A., Kanudia, A., Loulou, R., van Regemorter, D., \& Vaillancourt, K. (2004). The EFDA World Model. Final Report prepared for EFDA, ORDECSYS, HALOA/KANORS and KUL.

14. Barreto, L. (2001). Technological Learning in Energy Optimisation Models and the Deployment of Emerging Technologies. Ph.D. Thesis No 14151. Swiss Federal Institute of Technology Zurich.

15. Rafaj, P., Kypreos, S., \& Barreto, L. (2005). Flexible carbon mitigation policies: analysis with a global multi-regional MARKAL model. In A.
Haurie \& L. Viguier (Eds.), Coupling climate and economic dynamics. Dordrecht: Kluwer Academic Publishers. ISBN 1-4020-3424-5.

16. Messner, S., \& Schrattenholzer, L. (2000). MESSAGE-MACRO: linking an energy supply model with a macroeconomic module and solving it iteratively. Energy, 25(3), 267-282.

17. Büeler, B. (1997). Solving an equilibrium model for trade of $\mathrm{CO}_{2}$ emission permits. European Journal of Operational Research, 102(1997), 393-403.

18. Bahn, O., Barreto, L., Büeler, B., \& Kypreos, S. (1997). A Multiregional MARKAL-MACRO Model to Study an International Market of $\mathrm{CO}_{2}$ Emission Permits, PSI Report No. 97-09, Paul Scherrer Institute, Villigen, Switzerland.

19. Negishi, T. (1972). General equilibrium theory and international trade. Amsterdam: North-Holland Publishing Company.

20. Rutherford, T. (1999). Sequential joint maximization. In J. Weyant (Ed.), Energy and environmental policy modeling. Kluwer: International Series in Operations Research and Management Science.

21. Manne, A., Mendelsohn, R., \& Richels, R. (1995). MERGE: a model for evaluating regional and global effects of GHG reduction policies. Energy Policy, 23(1), 17-34.

22. Kypreos, S. (1996). The Markal-macro model and the climate change. PSI Report Nr 96-14, Paul Scherrer Institut (PSI), July 1996, Villigen, Switzerland.

23. Remme, U., \& Markus, B. (2006). Documentation of the TIMESMACRO model. http://www.etsap.org.

24. Kypreos, S. (2006). An algorithm to decompose the global MARKAL-MACRO (GMM) and TIMES models. working document for ETSAP. Switzerland: PSI.

25. Mousavi, B. (2014). Global decarburization pathways: contribution of different options in $\mathrm{CO}_{2}$ reduction: TIAM-MACRO, Decomposition analysis. http://www.iea-etsap.org/web/ Copenhagen_Nov2014.asp. 